CERN-TH/95-214

BI-TP 95/30

\title{
CHARMONIUM COMPOSITION AND NUCLEAR SUPPRESSION
}

\author{
D. Kharzeev and H. Satz \\ Theory Division, CERN, CH-1211 Geneva, Switzerland \\ and \\ Fakultät für Physik, Universität Bielefeld, D-33501 Bielefeld, Germany
}

\begin{abstract}
:
We study charmonium production in hadron-nucleus collisions through the intermediate next-to-leading Fock space component $\left|(c \bar{c})_{8} g\right\rangle$, formed by a colour octet $c \bar{c}$ pair and a gluon. We estimate the size of this state and show that its interaction with nucleons accounts for the observed charmonium suppression in nuclear interactions.
\end{abstract}

CERN-TH/95-214

BI-TP 95/30

August 1995 
Charmonium production in hadronic collisions inherently involves different energy and time scales; for an extensive recent treatment, see [1]. The first step is the creation of a heavy $c \bar{c}$ pair, e.g., by gluon fusion; this takes place in a very short time, $\tau_{\text {pert }} \simeq m_{c}^{-1}$. The pair is in a colour octet state; to neutralise its colour and yield a resonance state of $J / \psi$ quantum numbers, it has to absorb or emit an additional gluon (Fig. 1). The time $\tau_{8}$ associated to this process is determined by the virtuality of the intermediate $c \bar{c}$ state. In the rest frame of the $c \bar{c}$, it is approximately [2]

$$
\tau_{8} \simeq \frac{1}{\sqrt{\Delta}}
$$

where $\Delta \equiv\left[(p+k)^{2}-m_{c}^{2}\right]=2 p k$. For massless quarks, this gives the familiar $1 / k_{T}$ for the time uncertainty associated with gluon emission/absorption; for charm quarks of (large) mass $m_{c}$, we get

$$
\tau_{8} \simeq \frac{1}{\sqrt{2 m_{c} k_{0}}}
$$

where $k_{0}$ is the energy of the additional gluon. If it is sufficiently soft, $\tau_{8}>\tau_{\text {pert }}$. For $J / \psi$ production at mid-rapidity of a nucleon-nucleon collision, the colour neutralisation time becomes

$$
t_{8} \simeq \tau_{8}\left[1+\left(P_{A} / 2 m_{c}\right)^{2}\right]^{1 / 2}
$$

in the rest frame of target or projectile nucleon, with $P_{A}$ denoting the momentum of the $c \bar{c}$ pair in this frame. As seen from the nucleon, colour neutralisation of fast $c \bar{c}$ pairs will thus take a long time. Equivalently, a fast $c \bar{c}$ travels in the time $t_{8}$ a long distance,

$$
d_{8} \simeq \tau_{8}\left(P_{a} / 2 m_{c}\right),
$$

in the rest frame of the nucleon. On the basis of the process shown in Fig. 1, this seems to imply the existence of a coloured $c \bar{c}$ state of well-defined quantum numbers over times or distances much greater than the confinement scale of about $1 \mathrm{fm}$ (corresponding to $\Lambda_{\mathrm{QCD}}^{-1}$ with $\Lambda_{\mathrm{QCD}} \simeq 0.2 \mathrm{GeV}$ ). This problem is particularly evident for $J / \psi$ production at low transverse momentum, for which the additional gluon has to be quite soft; in this case, however, perturbative arguments become in any case questionable. The problem seems avoidable for production at sufficiently high $p_{T}$, i.e., for large enough $k_{0}$. Hence the colour singlet model $[3,4]$, which treats the complete colour singlet formation process in Fig. 1 within perturbative QCD, was usually restricted to high $p_{T}$ production. The low $p_{T}$ problem was until now usually "solved" by noting that inclusive $J / \psi$ production occurs in the colour field of the collision, leaving the form of the colour neutralisation unspecified ("colour evaporation" $[5,6]$ ).

Recent data [7] have shown, however, that also at high $p_{T}$ non-perturbative longtime features seem to be essential for charmonium production $[1,8]$. The most important outcome of these studies (see [9] for a recent review) is that higher Fock space components of charmonium states play a dominant role in their production. We thus decompose the $J / \psi$ state $|\psi\rangle$

$$
|\psi\rangle=a_{0}\left|(c \bar{c})_{1}\right\rangle+a_{1}\left|(c \bar{c})_{8} g\right\rangle+a_{2}\left|(c \bar{c})_{1} g g\right\rangle+a_{2}^{\prime}\left|(c \bar{c})_{8} g g\right\rangle+\ldots
$$


into a pure $c \bar{c}$ colour singlet component $\left({ }^{3} S_{1}\right)$, into a component consisting of $c \bar{c}$ colour octet $\left({ }^{1} S_{0}\right.$ or $\left.{ }^{3} P_{J}\right)$ plus a gluon, and so on. The higher Fock space coefficients correspond to an expansion in the relative velocity $v$ of the charm quarks. For the wave function of the $J / \psi$, the higher components thus correspond to (generally small) relativistic corrections. For $J / \psi$ production, however, their role can become decisive. While in short-time production the lowest component is the most important, in long-time processes the next higher term becomes dominant.

Analogous decompositions hold for the other charmonium states [1,8]. In all cases, the first higher Fock space state consists of a colour octet $c \bar{c}$ plus a gluon. For the $\psi^{\prime}$, the next-to-leading terms again contain a colour octet $\left({ }^{3} P_{J}\right.$ or $\left.{ }^{1} S_{0} c \bar{c}\right)$ plus a gluon; for the $\chi$ 's, a ${ }^{3} S_{1}$ colour octet $c \bar{c}$ is combined with a gluon.

This sheds some light onto the unspecified colour evaporation process. When the colour octet $c \bar{c}$ leaves the field of the nucleon in which it was produced, it will in general neutralise its colour by combining non-perturbatively with an additional collinear gluon, thus producing the $(c \bar{c})_{8} g$ component of the $J / \psi$ or the other charmonium states (Fig. 2). A necessary prerequisite for this is the small size of the $c \bar{c}$, due to the heavy quark mass; it is only because of this that the soft gluon interacts with the $c \bar{c}$ as a colour octet and not with the individual quarks. After the "relaxation time" $\tau_{8}$, the $(c \bar{c})_{8} g$ will then absorb the accompanying gluon to revert to the dominant $(c \bar{c})_{1}$ charmonium mode (Fig. 3 ). Note that we are here considering those $c \bar{c}$ pairs which will later on form charmonia. The $(c \bar{c})_{8}$ could as well neutralise its colour by combining with a light quark-antiquark pair, but this would result predominantly in open charm production.

The production of charmonia in a kinematic regime involving long time scales thus implies the production of the composite and hence extensive state $(c \bar{c})_{8} g$. Its intrinsic transverse size $r_{8}$ can be estimated through the uncertainty in the transverse momentum induced in the charm quark when it absorbs the accompanying gluon to go from $\left|(c \bar{c})_{8} g\right\rangle$ to the basic Fock component $\left|(c \bar{c})_{1}\right\rangle$ (Fig. 3). From the non-relativistic form for heavy quarks,

$$
\frac{p^{2}}{2 m_{c}} \simeq k_{0},
$$

where $p$ is the quark transverse momentum in the $(c \bar{c})_{8} g \mathrm{cms}$, we obtain from the lowest allowed gluon energy $k_{0}=\Lambda_{\mathrm{QCD}}$ the intrinsic size

$$
r_{8} \simeq \frac{1}{\sqrt{2 m_{c} \Lambda_{\mathrm{QCD}}}} \simeq 0.25 \mathrm{fm} .
$$

Since this size is determined only by the $(c \bar{c})_{8} g$ composition of the next-to-leading Fock space state and the gluon momentum cut-off in confined systems, it is the same for all charmonium states. In general, the time and momentum uncertainty would be given by the binding energy and the size of the state, i.e., for the $J / \psi$ by the mass difference $\epsilon_{0}=2 M_{D}-M_{\psi} \simeq 0.64 \mathrm{GeV}$ between it and open charm. This would imply different sizes for the $(c \bar{c})_{8} g$ component of different resonance states; for a related discussion, see [10]. In the case of all charmonium and bottonium states, however, the common confinement cut-off $\sqrt{2 m_{Q} \Lambda_{\mathrm{QCD}}}$ is more stringent and hence the relevant one. 
We now want to study the effect of these considerations on $J / \psi$ production in proton-nucleus collisions. For low $P_{T}$ production, with $k_{0} \simeq \Lambda_{\mathrm{QCD}}$ in Eq. (2), $\tau_{8}$ would exceed the size of the even heavy nuclei for $J / \psi$ 's of sufficiently high lab momenta. Maintaining an approach based on the colour singlet model would thus require a colour octet $c \bar{c}$ to pass through the entire nuclear medium [2][11-14]. The composition of the colour-neutralising cloud needed for this was so far undetermined, and hence estimates for the resulting cross sections were generally obtained by assuming such a dressed $(c \bar{c})_{8}$ to be of hadronic size $[2,11,15]$. It was also left open why interactions with the surrounding cloud would not destroy the spatial and quantum number structure of the $(c \bar{c})_{8}$. Quarkonium production through higher Fock space components now provides a specific description of the $(c \bar{c})_{8}$ passage through the nucleus. The system leaves the nucleon in whose field it was formed as a colour singlet $(c \bar{c})_{8} g$ and continues as such through the nuclear medium. The size of this charmonium state $(c \bar{c} g) \equiv\left|(c \bar{c})_{8} g\right\rangle$ is given by Eq. (7) and is thus essentially that of a $J / \psi$. Also its interaction with hadrons is similar to the $J / \psi$-hadron interaction [16], but with two important distinctions. The gluon exchanged between the two colliding systems will now couple predominantly to the gluon or to the $(c \bar{c})_{8}$ component of the $(c \bar{c} g)$. Since both are colour octets, in contrast to the colour triplet components of the $J / \psi$, the coupling is correspondingly enhanced by a factor $9 / 4$. Such an interaction will render the $(c \bar{c})_{8} g$ system coloured. Due to the repulsive one-gluon exchange interaction, the colour octet $\left(c \bar{c}_{8}\right)$ is not bound, in contrast to the colour singlet $(c \bar{c})_{1}$. Moreover, the probability of the $(c \bar{c})_{8}$ encountering another collinear gluon to again form a colour singlet system of $J / \psi$ quantum numbers is minimal. Hence any $(c \bar{c} g)$ interaction will generally lead to its break-up, so that there is no threshold factor. The cross section for $(c \bar{c} g)$-hadron interactions is thus just the geometric $J / \psi$-hadron cross section, $\sigma_{\psi N}$, increased by the enhanced coupling,

$$
\sigma_{(c \bar{c} g) N}=\frac{9}{4} \sigma_{\psi N}
$$

Short-distance QCD calculations [16] lead to $\sigma_{\psi N} \simeq 2.5-3 \mathrm{mb}$, in good agreement with photoproduction data [17]. From this we get $\sigma_{(c \bar{c} g) N} \simeq 6-7 \mathrm{mb}$. Because of the general nature of the arguments leading to this value, it holds equally for the interactions of the next-to-leading Fock space components of $\psi^{\prime}$ and $\chi$ with hadrons.

This cross section, while larger than the high energy $J / \psi$-hadron cross section by about a factor two, is very much smaller than the hadronic value of $20-50 \mathrm{mb}$ previously assumed for the colour octet $c \bar{c}$ passing through the nucleus $[2,11,15]$. This has immediate consequences. The mean free path of the $(c \bar{c} g)$ in nuclear matter, $\lambda_{(c \bar{c} g)}=$ $1 / n_{0} \sigma_{(c \bar{c} g) N} \simeq 10 \mathrm{fm}$ is larger than the radius of even the heaviest nuclei. Moreover, since the $c \bar{c}$ combines with an already existing collinear gluon, there is no coherence length associated with any $(c \bar{c} g)$ formation. Hence shadowing $[18,19]$ is excluded as dominant quarkonium suppression mechanism in $p-A$ collisions.

In passing through the nucleus, the small physical state $(c \bar{c} g)$ will interact incoherently with the nucleons along its trajectory. The charmonium production probability on nuclei relative to that on nucleons, valid for $J / \psi, \chi$ and $\psi^{\prime}$ production, thus becomes

$$
S_{A}=\exp \left\{-n_{0} \sigma_{(c \bar{c} g) N} L\right\}
$$


where $L$ denotes the length of the path through nuclear matter of standard density $n_{0}=0.17 \mathrm{fm}^{-3}$, and $\sigma_{(c \bar{c} g) N} \simeq 6-7 \mathrm{mb}$ is the inelastic $(c \bar{c} g)$-nucleon cross section obtained above.

With Eq. (9) we have derived the Gerschel-Hüfner fit [20], introduced as a phenomenological description of hadron-nucleus data on $J / \psi$ production. In [20], it was attempted to interpret the cross section entering in Eq. (9) as the physical $J / \psi$-hadron cross section, which led to theoretical as well as experimental problems. The fit value was 5 - $7 \mathrm{mb}$; both short distance QCD [16] and photoproduction experiments [17] give a $J / \psi$-hadron cross section smaller by at least a factor two. Moreover, $p-A$ data $[21,22]$ lead to exactly the same suppression of $\psi^{\prime}$ and $J / \psi$ production for all $A$, with an $A$-independent production ratio $\psi^{\prime} /(J / \psi) \simeq 0.15$. Since the geometric size of the ground state $J / \psi$ and the next radial excitation differ by more than a factor four, an equal suppression contradicts the interaction of fully formed physical resonances. We find here that all aspects of the observed suppression arise naturally in charmonium production through the next higher Fock space component $(c \bar{c} g)$. A composite state $(c \bar{c} g)$, which is of the same size for all charmonia, passes through the nuclear medium and hence leads to equal $\psi^{\prime}$ and $J / \psi$ suppression. The value of the cross section $\sigma_{(c \bar{c} g) N}$ thus obtained is in good agreement with that obtained from a fit to the data [20].

The same argumentation also provides the suppression of bottonium production in $p-A$ collisions. The radius of the $(b \bar{b})_{8} g$ state is (see Eq. (7)) a factor $\sqrt{m_{c} / m_{b}} \simeq \sqrt{3}$ smaller than that of the $(c \bar{c} g)$, so that $\sigma_{(b \bar{b} g) N} \simeq(1 / 3) \sigma_{(c \bar{c} g) N} \simeq 2 \mathrm{mb}$.

To illustrate how well both $J / \psi$ and $\Upsilon$ production in $p-A$ collisions are described by this scenario, we show in Fig. 4 recent high energy data at $\sqrt{s} \simeq 20$ [22], 30 [23] and $40 \mathrm{GeV}[21]$. With an average path length $L_{A}=(3 / 4)[(A-1) / A]\left[1.12 A^{1 / 3}\right]$ we obtain excellent agreement for $\sigma_{(c \bar{c} g) N} \simeq 6 \mathrm{mb}$ and $\sigma_{(b \bar{b} g) N} \simeq 2 \mathrm{mb}$. In [20] it was shown that $J / \psi$ production data from $\pi-A$ collisons lead to very similar values for the cross section in Eq. (9).

With quarkonium suppression in hadron-nucleus collisions thus accounted for in terms of interaction between $(Q \bar{Q})_{8} g$ states and nucleons, we can now also consider nucleus-nucleus interactions. For collision energies of $\sqrt{s} \simeq 20 \mathrm{GeV}$, the centers of the two colliding nuclei have at time $t_{8}$ separated in the cms by about $5 \mathrm{fm}$. With the nuclei Lorentz-contracted to a thickness of about $0.5 \mathrm{fm}$ or less, this means that a $J / \psi$ produced at mid-rapidity in the cms has experienced in its early phase an effect corresponding to that obtained by superimposing the passages through the two nuclei [20]. Hence the charmonium suppression now is given by

$$
S_{(A-B)}=\exp \left\{-n_{0} \sigma_{(c \bar{c} g) N}\left(L_{A}+L_{B}\right)\right\}
$$

again in accord with the phenomenological fit of [20]. The path length $L=L_{A}+L_{B}$ in Eq. (10) varies with impact parameter and hence with the associated transverse energy $E_{T}$ produced in the collision. A relation between $L$ and $E_{T}$ can thus be obtained through a detailed study of the collision geometry [24]. An alternative is given by determining $L$ through the broadening of the average transverse momentum of $J / \psi$ or Drell-Yan dileptons, since this broadening also depends on the average path length [25]. In Fig. 5 
we show the resulting values [22], normalised to the $(c \bar{c} g)$ suppression of Eqs. (8) and (9), for both $p-A$ and $S-U$ data. We conclude that also the $J / \psi$ suppression observed so far in $S-U$ collisions is completely accounted for by $(c \bar{c} g)$ suppression in standard nuclear matter.

The comparison of $J / \psi$ and $\psi^{\prime}$ production in nucleus-nucleus collisions provides a test for the presence of a medium in the later stages. As long as the interaction leading to charmonium suppression is determined by the $(c \bar{c} g)$ state, $J / \psi$ and $\psi^{\prime}$ must be equally suppressed. To make $J / \psi$ and $\psi^{\prime}$ suppression different, the medium must see the fully formed resonances and distinguish between them. In Fig. 5 we have included also the $\psi^{\prime}$ suppression divided by the $(c \bar{c} g)$ suppression $(8)[26,22]$. It is evident that in $S-U$ collisions $J / \psi$ and $\psi^{\prime}$ are not affected equally, the $\psi^{\prime}$ being much stronger suppressed. This establishes the presence of a medium at a time late enough for fully formed charmonium resonances to exist. On the other hand, this medium breaks up only the $\psi^{\prime}$, leaving the $J / \psi$ unaffected. It was shown [16] that interactions with hadrons in the range of present collision energies cannot dissociate a $J / \psi$, while for the much more loosely bound $\psi^{\prime}$ this is readily possible. We therefore conclude that the medium probed by charmonium production in present $S-U$ collisions is of hadronic nature, i.e., confined; it could consist either of stopped nucleons or of secondary hadrons produced in the collision [27]. Note that we include the $\psi^{\prime}$ data as function of $L$ in Fig. 5 simply to show the additional suppression present in this case. It is not at all clear that $L$ is a meaningful variable for the effect of such a confined environment on charmonia. We also note that the appearent absence of any effect of this medium on the observed $J / \psi$, even though this is to about $40 \%$ produced through $\chi$ decay, is in accord with the short distance QCD form of the $\chi$-hadron cross section [28].

To establish colour deconfinement, either in equilibrium or pre-equilibrium systems, nucleus-nucleus collisions have to produce a $J / \psi$ suppression beyond that given by Eq. (10), i.e., beyond that found in $p-A$ collisions [20], and different from $\psi^{\prime}$ suppression. If such an additional suppression were found, the results for inelastic $J / \psi$-hadron collisions obtained from short distance QCD [2] would rule out a confined medium, and the difference in $J / \psi$ and $\psi^{\prime}$ suppression would exclude $(c \bar{c} g)$ interactions as a source.

In summary: we study quarkonium production in hadron-nucleus collisions through the intermediate next-to-leading Fock space state consisting of a colour octet $c \bar{c}$ and a gluon. We estimate the inelastic $(c \bar{c} g)$-nucleon cross section and use this to

- exclude shadowing as main origin for the quarkonium suppression observed in hadron-nucleus interactions;

- derive the Gerschel-Hüfner fit describing such suppression, both for $c \bar{c}$ and $b \bar{b}$ states;

- conclude that the $J / \psi$ suppression observed in $S-U$ collisions is fully accounted for by early $(c \bar{c} g)$ interactions with standard nuclear matter;

- conclude that the stronger $\psi^{\prime}$ suppression found in $S-U$ collisions is due to an additional confined medium present at a later stage.

It will be interesting to see if the results of forthcoming $P b-P b$ collisions can provide first indications for deconfinement - in particular, if they show a stronger $J / \psi$ dissociation than accounted for by $(c \bar{c} g)$ interactions. 


\section{Acknowledgements}

We thank A. Capella, C. Gerschel, A. Kaidalov, C. Lourenco and M. Mangano

for stimulating and helpful discussions. The support of the German Research Ministry BMFT under contract 06 BI 721 is gratefully acknowledged.

\section{References}

1) G. T. Bodwin, E. Braaten and G. P. Lepage, Phys. Rev. D 51 (1995) 1125

2) D. Kharzeev and H. Satz, Z. Phys. C 60 (1993) 389.

3) C. H. Chang, Nucl. Phys. B 172 (1980) 425;

E. L. Berger and D. Jones, Phys. Rev. D 23 (1981) 1521;

R. Baier and R. Rückl, Phys. Lett. B 102 (1981) 364 and Z. Phys. C 19 (1983) 251.

4) For a recent survey, see G. A. Schuler, "Quarkonium Production and Decays", CERN-TH.7174/94, 1994, to appear in Physics Reports.

5) M. B. Einhorn and S. D. Ellis, Phys. Rev. D12 (1975) 2007;

H. Fritzsch, Phys. Lett. 67B (1977) 217;

M. Glück, J. F. Owens and E. Reya, Phys. Rev. D17 (1978) 2324;

J. Babcock, D. Sivers and S. Wolfram, Phys. Rev. D18 (1978) 162.

6) For a recent survey, see R. V. Gavai et al., "Quarkonium Production in Hadronic Collisions", CERN-TH.7526/94, 1994, to appear in Int. J. Mod. Phys. A.

7) See e.g. V. Papadimitriou (CDF), "Production of Heavy Quark States at CDF", Preprint Fermilab-Conf-95-128 E, March 1995;

L. Markosky (D0), "Measurements of Heavy Quark Production at D0", Preprint Fermilab-Conf-95-137 E, May 1995.

8) E. Braaten and S. Fleming, Phys. Rev. Lett. 74 (1995) 3327.

9) M. L. Mangano, "Phenomenology of Quarkonium Production in Hadronic Collisions", CERN-TH/95-190.

10) A. H. Mueller, Nucl. Phys. B 415 (1994) 373.

11) G. Piller, J. Mutzbauer and W. Weise, Z. Phys. A 343 (1992) 247, and Nucl. Phys. A 560 (1993) 437.

12) S. Gavin and J. Milana, Phys. Rev. Lett. 68 (1992) 1834.

13) R. Wittmann and U. Heinz, Z. Phys. C 59 (1993) 77.

14) K. Boreskov et al., Phys. Rev. D47 (1993) 919.

15) J. Dolejši and J. Hüfner, Z. Phys. C 54 (1992) 489.

16) D. Kharzeev and H. Satz, Phys. Lett. B 334 (1994) 155.

17) S. D. Holmes, W. Lee and J. E. Wiss, Ann. Rev. Nucl. Part. Sci. 35 (1985) 397.

18) S. Gupta and H. Satz, Z. Phys. C 55 (1992) 391.

19) D. Kharzeev and H. Satz, Phys. Lett. B 327 (1994) 361.

20) C. Gerschel and J. Hüfner, Z. Phys. C 56 (1992) 171.

21) D. M. Alde et al., Phys. Rev. Lett. 66 (1991) 133;

D. M. Alde et al., Phys. Rev. Lett. 66 (1991) 2285. 
22) C. Lourenco (NA38/51), "Recent Results on Dimuon Production from the NA38 Experiment", CERN-PPE/95-72, May 1995, and Doctorate Thesis, Universidade Técnica de Lisboa, Portugal, January 1995.

23) L. Fredj (NA38/51), Doctorate Thesis, Université de Clermont-Ferrand, France, September 1991.

24) R. Salmeron, Nucl. Phys. B 389 (1993) 301.

25) C. Baglin et al., Phys. Lett. B 268 (1991) 453.

26) C. Baglin et al., Phys. Lett. B 345 (1995) 617.

27) C.-Y. Wong, "Suppression of $\psi^{\prime}$ and $J / \psi$ in High Energy Heavy Ion Collisions", Oak Ridge Preprint ORNL-CTP-95-04, June 1995.

28) D. Kharzeev and H. Satz, "Colour Deconfinement and Quarkonium Dissociation", Preprint CERN-TH/95-117, May 1995; to appear in R. C. Hwa (Ed.), Quark-Gluon Plasma II, World Scientific Publ. Co., Singapore.

\section{Figure Captions}

Fig. 1: $J / \psi$ production through gluon fusion.

Fig. 2: $J / \psi$ production via $(c \bar{c} g)$ colour singlet.

Fig. 3: Transition from $(c \bar{c} g)$ colour singlet to $c \bar{c}$ colour singlet.

Fig. 4: $J / \psi$ and $\Upsilon$ suppression in $p-A$ collisions; data [21-23] are compared to $(c \bar{c} g)$ suppression (Eq. (9)) in nuclear matter and to the corresponding form for $(b \bar{b} g)$.

Fig. 5: $J / \psi, \psi^{\prime}$ and $\Upsilon$ suppression in $p-A$ and $S-U$ collisions, normalised to $(c \bar{c} g)$ and $(b \bar{b} g)$ suppression in nuclear matter. 
This figure "fig1-1.png" is available in "png" format from: http://arxiv.org/ps/hep-ph/9508276v1 
This figure "fig1-2.png" is available in "png" format from: http://arxiv.org/ps/hep-ph/9508276v1 
This figure "fig1-3.png" is available in "png" format from: http://arxiv.org/ps/hep-ph/9508276v1 